\title{
The role of long non-coding RNA GAS5 in cancers
}

This article was published in the following Dove Press journal:

Cancer Management and Research

Jiali Ji $\mathrm{i}^{1}$

\section{Xiaolan Dai ${ }^{2}$ \\ Sai-Ching Jim Yeung ${ }^{3}$ \\ Xuexin $\mathrm{He}^{\prime}$}

'Department of Medical Oncology, The 2nd Affiliated Hospital of Zhejiang University, Hangzhou, Zhejiang, People's Republic of China; ${ }^{2}$ Department of Pharmacy, School of Medicine, Shantou University, Shantou, Guangdong, People's Republic of China; ${ }^{3}$ Division of Internal Medicine, The University of Texas MD Anderson Cancer Center, Houston, TX, USA
Correspondence: Xuexin He Department of Medical Oncology, The 2nd Affiliated Hospital of Zhejiang University, Hangzhou, Zhejiang, People's Republic of China

Tel +8618329139569

Email xuexinhe@zju.edu.cn

\begin{abstract}
Long non-coding RNAs (lncRNAs) have shown potential as a biomarker in the diagnosis and prognosis in multiple cancers. LncRNAs are dysregulated in various cancers, playing either oncogenic or tumor suppressive roles. Emerging evidences have proved that the growth arrest-specific 5 (GAS5) lncRNA can function as a tumor suppressor in several cancers. LncRNA GAS5 is downregulated in many types of cancer, regulating cellular processes such as cell proliferation, apoptosis and invasion. The low level of GAS5 expression often elevates capacity of proliferation and predicts poorer prognosis in some cancers. This review aims to summarize the recent published literature on the biogenesis, regulation mechanism and function of GAS5 in different types of cancers and explore its potential for cancer diagnosis, prognosis and treatment.
\end{abstract}

Keywords: lncRNA, GAS5, tumor suppressor, tumor

\section{Introduction}

The incidence of cancers has been increasing over the years. ${ }^{1}$ It is universally acknowledged that cancer is a major health issue. Early detection of cancer can greatly increase the probability for curative therapy. Therefore, the search for new diagnostic and prognostic biomarkers and effective therapies goes on.

Advances in high-throughput sequencing have led to the discovery of novel non-coding RNAs (ncRNAs) in recent years. Long non-coding RNAs (lncRNAs) are a group of RNA molecules longer than 200 nucleotides in length that are distinct from known categories of structural RNAs. ${ }^{2}$ LncRNAs, initially thought to be non-functional, ${ }^{3}$ were found to play important roles in many human diseases, especially in malignancies. ${ }^{4-10}$ Increasing evidence suggests that some lncRNAs have important roles in carcinogenesis and cancer progression, and may serve as diagnostic and/or prognostic biomarkers for some cancers. ${ }^{11}$

The growth arrest-specific 5 (GAS5) gene can encode ncRNAs of various sizes including a lncRNA. ${ }^{12}$ GAS5 is a tumor suppressor gene located at chromosome 1q25 and was first discovered in 1988 by screening highly expressed genes in growth-arrest cells. ${ }^{13,14}$ Downregulation of GAS5 expression is observed in many cancers. Clinical outcomes such as lymph node metastasis, tumor recurrence and overall survival are correlated with the expression of GAS5 in various cancers. ${ }^{15-17}$

The level of GAS5 expression regulates apoptosis, proliferation, invasion, epithelial-mesenchymal transition and metastasis of cancer cells, ${ }^{18-21}$ and may have prognostic value in various clinical scenarios. ${ }^{22-25}$ Although the precise molecular mechanism remains unclear, lncRNA GAS5 certainly plays an important 
role in carcinogenesis and tumor progression. In our review, we will discuss the potential molecular mechanisms, recent research and clinical assessment of lncRNA GAS5 in cancer. An improved understanding of IncRNA GAS5 in cancer may provide new insights and inspiration for future clinical application.

\section{Gene structure of GAS5}

GAS5 belongs to the 5'-terminal oligopyrimidine (5'-TOP) gene family, which includes all ribosomal proteins, protein synthesis elongation factors as well as many genes without ribosome-related functions. ${ }^{26}$ GAS5 is a non-proteincoding gene located at chromosome 1q25.1 [molecular location: Chromosome 1, NC_000001.11 (173,863,899 to $173,868,882$, complement)] and composed of 12 exons which constitute a short open reading frame that does not encode a protein. Some parts of the introns with highly conserved regions are loci of some small nucleolar RNAs (snoRNAs). The essential biological activities of GAS5 may depend, at least in part, on introns that encode multiple snoRNAs ${ }^{27}$ and on lncRNA GAS5/snoRNA-derived PIWI-interacting RNA (piRNA). ${ }^{12}$

\section{Molecular mechanisms of IncRNA GAS5}

About 7 years ago, 4 main types of mechanisms of action for lncRNA were summarized: 1) signals for transcription, 2) decoys for transcription factors, 3) guides of transcription factors and 4) scaffolds for protein complexes that epigenetically modify chromatin. ${ }^{28}$ More recently, the mechanisms of action were expanded to include: 1) lncRNA transcriptiondependent activation or repression of neighboring genes, 2) inter-chromosomal interactions, 3) formation of nuclear structures (ie, paraspeckles) or R-loops, 4) lncRNAs acting as sponges of miRNAs, 5) regulating post-transcriptional mRNA decay and 6) regulating the cellular localization of RNA-binding proteins or DNA-binding proteins. ${ }^{29}$

The biological processes regulated by GAS5 are summarized in Figure 1. Specifically for GAS5, review of the literature supports the following mechanisms:

\section{Transcriptional regulation through acting as a decoy}

Glucocorticoid hormones can accelerate catabolism, modulate the immune response and cell survival when the body is under internal or external stress. ${ }^{30,31}$ Kino et al discovered that IncRNA GAS5 can act as a decoy for glucocorticoid receptor (GR). ${ }^{32}$ LncRNA GAS5 can bind to the DNA-binding domain of GR and titrate down the amount of GR that is available to bind glucocorticoid response elements (GREs) in the genomic DNA. Although this RNA and protein interaction between IncRNA GAS5 and GR blocks the binding between GRE and GR in the context of growth arrest and starvation, its relevance in cancer cells is unclear.

\section{Transcriptional regulation through histone methylation/demethylation}

In bladder cancer cells, lncRNA GAS5 can directly interact with transcription factor E2F4 and increase its binding to the promoter region of enhancer of zeste homolog 2 (EZH2), a histone-lysine N-methyltransferase gene. ${ }^{33}$ Consequently, transcription of miR-101 was upregulated and bladder cancer cell growth was suppressed. LncRNA

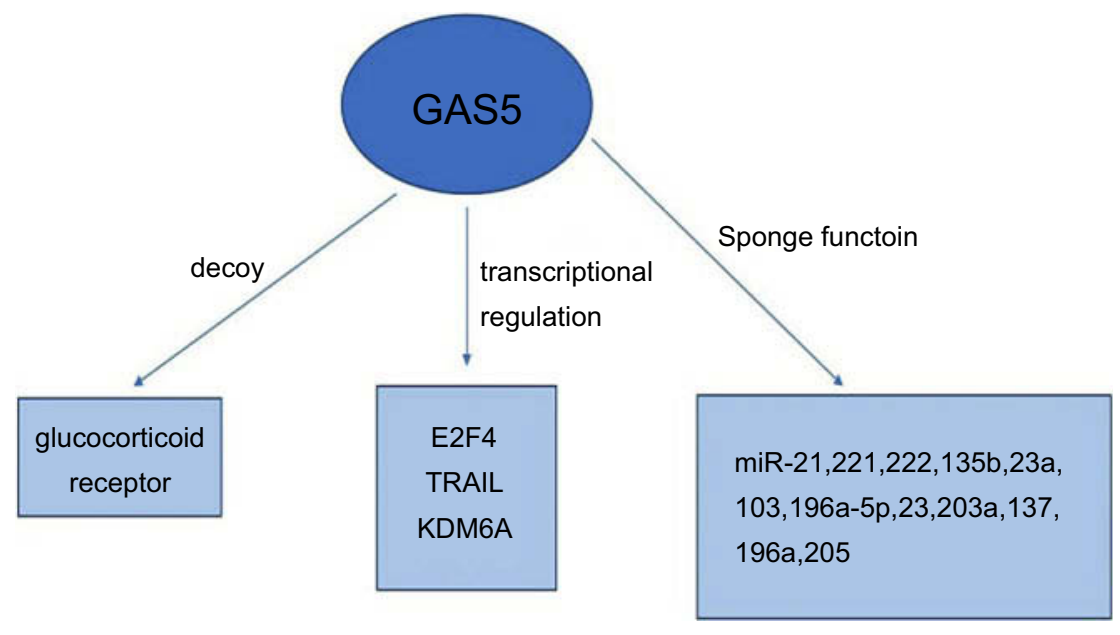

Figure I The biological processes regulated by LncRNA GAS5. 
GAS5 can also indirectly regulate gene expression through its small RNA derivatives. For instance, GAS5-derived piRNA induces histone $\mathrm{H} 3$ lysine 4 methylation and histone $\mathrm{H} 3$ lysine 27 demethylation and resulted in increased transcription of tumor necrosis factor-related apoptosisinducing ligand (TRAIL), a proapoptotic protein. ${ }^{12}$ The GAS5-derived piRNA binds to PIWIL1/4 proteins, which interact with WDR5 to recruit hCOMPASS-like complexes containing MLL3 and UTX (KDM6A), constituting a molecular mechanism relevant to tumor suppression. Nevertheless, it is very challenging to determine which biological effect of GAS5 is attributable to the intact lncRNA, its small RNA derivatives or both.

\section{Mirna sponge function}

GAS5 can function as a competing endogenous RNA (ceRNA) to regulate signaling pathways and biological functions. Bioinformatics analysis of complementary regions of GAS5 to microRNAs identified 690 candidates, among which 234 microRNAs showed statistically significant binding. ${ }^{34}$ In the context of cancer, LncRNA GAS5 has been shown to bind to miR$21,{ }^{35,36}$ miR-221, ${ }^{37}$ miR-222, ${ }^{38,39}$ miR-135b, miR-23a and $\mathrm{miR}-103$ et al. ${ }^{40}$

miR-21: LncRNA GAS5 can suppress the expression of $m i R-21$, an oncogene in various solid tumors and lymphoma expression. ${ }^{35,36}$ Fibroblast factor1 (FGF1) is a mediator of the GAS5/miR-21 axis that regulates proliferation and apoptosis. ${ }^{41}$ In NSCLC, suppression of GAS5 expression can lead to chemoresistance to cisplatin because LncRNA GAS5 competes with phosphatase and tension homolog (PTEN) for miR-21 binding. ${ }^{42}$

miR-221: GAS5 can inhibit the expression of miR-221 and suppress cell proliferation, invasion and migration as well. ${ }^{43}$

miR-222: It can also suppress tumor by downregulating miR-222 resulted in reduced proliferation and invasion in glioma. $^{38}$

miR-135b: The overexpression of GAS5 can inhibit tumor growth and enhance radiosensitivity by downregulating $m i R-135 b$ expression in non-small cell lung cancer. ${ }^{44}$

miR-23a: In non-small cell lung cancer, GAS5 can directly interact with miR-23a and then reduce the expression of $m i R$ $23 a$ which can promote cell proliferation and invasion. ${ }^{45}$

miR-103: The aberrant expression of miR-103 was found to promote colorectal cancer by downregulating the expression of PTEN and regulate growth and invasion of endometrial cancer cells through downregulating the suppressor of TIMP-3. GAS5 can act as an inhibitor of miR-103 and then enhance the expression of PTEN to promote cancer apoptosis. ${ }^{40}$

miR-196a-5p: GAS5 was proved to suppress the proliferation, migration and invasion of glioma stem cells by binding to miR-196a-5p, an onco-miRNA contribute to glioma pathogenesis. ${ }^{46}$

miR-23: GAS5 can inhibit gastric cancer by regulating miR-23 as well. ${ }^{47}$

miR-203a: GAS5 can negatively regulate the expression of miR-203a and keep miR-203a away from its target gene TIMP2 which can inhibit cancer cell metastasis in osteosarcoma. $^{48}$

miR-137: In melanoma, GAS5 can positively regulate miR-137 and then promote cell proliferation, migration and invasion. ${ }^{49}$

miR-196a and miR-205: Overexpression of GAS5 can inhibit cell proliferation and invasion by binding to miR-196a and miR-205 in cervical cancer to suppress cell growth through aplasia Ras homolog member I (ARHI). ${ }^{50}$

\section{Signaling pathway associated with IncRNA GAS5}

It was reported that the inhibition of mammalian target of rapamycin (mTOR) pathway depends on GAS5. ${ }^{51,52}$ In prostate cancer, GAS5 was proved to inhibit cancer proliferation and progression by targeting miR-103 through the protein kinase B (AKT)/mTOR signaling pathway. ${ }^{53}$ Overexpression of GAS5 was proved to inhibit the miR222 expression and then suppressed cell proliferation in gastric cancer through phosphatase and tensin homolog (PTEN)/phosphorylated protein kinase B (Akt)/phosphorylated mammalian target of rapamycin (mTOR) pathway as well. ${ }^{54}$

GAS5 is a downstream target of Notch pathway in breast cancer. Notch1 can promote breast cancer cells proliferation by modulating $G A S 55^{55}$ In NSCLC cells, GAS5 overexpression was negatively correlated with the expression of EGFR pathway. ${ }^{56}$ GAS5 was associated with embryonic stem cells (CSCs) self-renewal by regulating NODAL signaling. ${ }^{57}$ In pancreatic cancer cells, GAS5 can inhibit the expression of miR-181c-5p and then prohibit cell chemoresistance through suppressing Hippo signaling. ${ }^{58}$ We summarized the function of GAS5 in various cancers in Figure 2. 


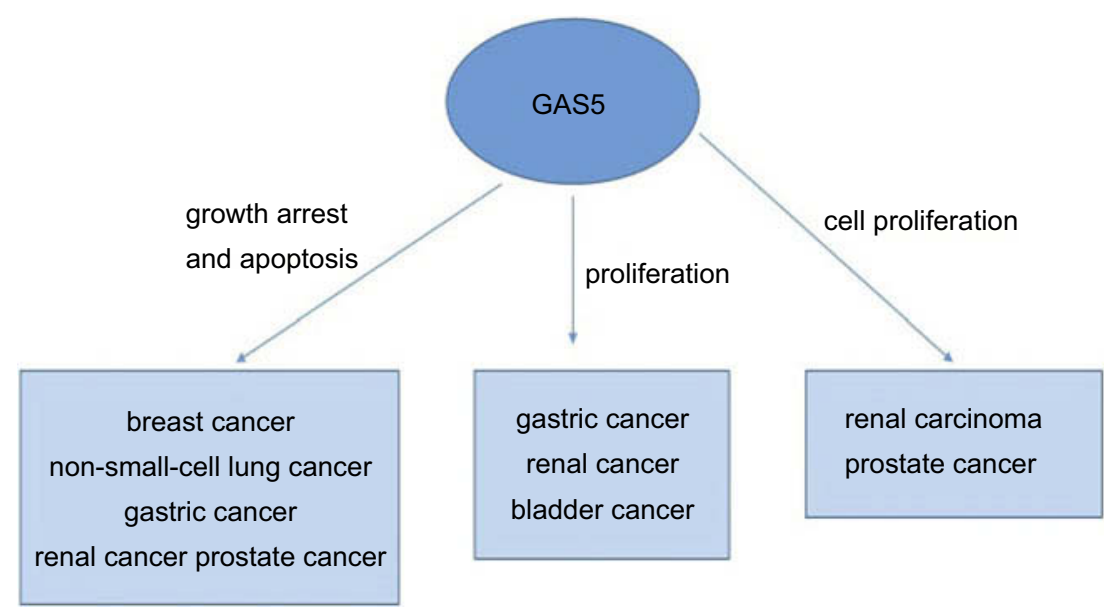

Figure 2 The function of LncRNA GAS5 in various cancers.

\section{Function in various cancers Gastrointestinal cancer}

Colorectal cancer (CRC) was the third common cancer in the world. It was reported that the expression of GAS5 in CRC was lower than those in normal tissue. Meanwhile, the low expression of GAS5 was significantly associated with large tumor size, low grade, advanced TNM stage, higher local recurrence rate and distant metastasis rate. ${ }^{43,59}$ The lower expression of GAS5 was associated with poor overall survival. Univariate and multivariate analysis further revealed that GAS5 was an independent prognostic factor for CRC. The overexpression of GAS5 can inhibit proliferation, migration and invasion in CRC. ${ }^{60}$ Yang et al have concluded that the overexpression of GAS5 can induce G0/G1 cell cycle arrest and apoptosis. ${ }^{61}$ Thus GAS5 may become a novel prognostic marker and a potential target for CRC in the future.

Previous studies have found that lncRNA GAS5 was downregulated in gastric cancer. Meanwhile, the downregulated level was significantly associated with larger tumor size and advanced pathologic stage. Patients with lower expression of GAS5 have poorer disease-free survival and overall survival than those with higher GAS5 expression. Vivo and vitro experiments further demonstrated that the GAS5 can upregulate proliferation and induce apoptosis in gastric cancer. ${ }^{17}$

It was reported that GAS5 expression was lower in pancreatic cancer than normal tissue. And overexpression of GAS5 can inhibit cell proliferation in pancreatic cancer by decreasing G0/G1 phase and increasing $\mathrm{S}$ phase. $^{21}$ Downregulation of GAS5 was associated with chemoresistance in pancreatic cancer as well. ${ }^{58}$
Moreover, the overexpression of GAS5 can inhibit pancreatic cancer cell tumorigenesis in vivo and suppress tumor metastasis. ${ }^{62}$

In hepatocellular carcinoma, overexpression of GAS5 could promote prognosis, suppress proliferation and invasion of hepatoma cells. Low expression of GAS5 was closely related with differentiation and poor overall survival. ${ }^{35,63}$

Wang et al have found that GAS5 was downregulated in esophageal cancer (EC) and could inhibit the growth of EC. ${ }^{64}$ However, $\mathrm{Li}$ et al reported that contrast to other cancers, the GAS5 was overexpressed in EC. GAS5 is proved to promote proliferation, metastasis and inhibited apoptosis in EC by regulating miR-301a. ${ }^{65}$ The concrete role and regulation function of GAS5 in EC still need further study.

\section{Malignant pleural mesothelioma (MPM)}

MPM is a malignant cancer with poor prognosis. Some lncRNAs have clinical significance like predicting metastasis and overall survival. Previous studies have shown that GAS5 expression is lower in MPM cells and its expression could be regulated by drugs inducing growth arrest in MPM. The high level of GAS5 could increase promoter activity. Moreover, GAS5 could control cell cycles in a glucocorticoid receptor-decoy way. As a result, GAS5 plays an important role in MPM and could be a targeted agent in the future. ${ }^{66}$

\section{Urological malignancies}

Renal cell carcinoma (RCC) is a common carcinoma with poor prognosis. Hui ping et al provide the first evidence that GAS5 expression was lower in RCC cells and the 
overexpression of GAS5 can inhibit cell proliferation, induce cell apoptosis, arrest cell cycle, progress cell death and inhibit invasion. Therefore, the decreased expression of GAS5 was associated with tumorigenesis and progression. ${ }^{67,68}$

In bladder cancer, accumulating studies have found that overexpression of $G A S 5$ can promote apoptosis in druginduced resistance. The upregulated expression of GAS5 can promote apoptosis by affecting GA induced apoptosis and inhibiting EZH2 transcription. ${ }^{33}$ The level of GAS5 was lower in bladder cancer tissues and the low expression of $G A S 5$ was positively related to higher pathological grades and could be a prognosis for disease-free survival of bladder cancer patients. Enhancement of GAS5 can also reduce the chemotherapy resistance to doxorubicin via $\mathrm{Bcl} 2$. $^{69}$

Prostate cancer is the most prevalent malignancy in male patients. Mouse xenograft models were used to explore the GAS5 effects on prostate cancer. Overexpression of GAS5 can significantly inhibit prostate cancer cell proliferation and tumor growth in vitro and vivo. ${ }^{53}$

\section{Breast cancer}

GAS5 expression is significantly downregulated in breast cancer cells. The overexpression of GAS5 can induce or facilitated apoptosis in breast cancer cells and produce an increase in sensitivity to treatments by several different pathways. ${ }^{18}$ Moreover, GAS5 can promote the apoptosis of triple negative and estrogen receptor-positive breast cancer cells. Pickard et al have reported that the use of mTOR inhibitors may enhance GAS5 levels to suppress cancer growth as well. ${ }^{70}$ The plasma level of GAS5 changes after surgery. The preoperative level of GAS5 can reflect the active degree of proliferation in breast cancer. Thus, the plasma GAS5 can be a biomarker to assess the prognosis evaluation after surgery. ${ }^{16}$ The expression of GAS5 was decreased in breast cancer cell from patients treated with trastuzumab and proved to contribute to trastuzumab resistance. ${ }^{71}$ As a result, GAS5 plays an important role in regulating breast cancer cells.

\section{Lung cancer}

Lung cancer is a common malignant cancer with the highest mortality in China. Compared with common tissues, the expression of GAS5 was decreased in non-small cell lung cancer (NSCLC). Moreover, GAS5 was declined in early stage before surgery compared with healthy control patients. $^{23}$ The overexpression of GAS5 can inhibit NSCLC cell proliferation, promote apoptosis and improve radiosensitivity of NSCLC cells. ${ }^{45}$ Decreased expression of GAS5 is correlated with advanced TNM stages and larger tumor size. It can regulate NSCLC chemoresistance to cisplatin-based therapy as well. ${ }^{42}$ Moreover, GAS5 was found to be overexpressed in epidermal growth factor receptor-tyrosine kinase inhibitors (EGFR-TKIs) sensitive cell lines compared with the resistant cell lines. $^{56}$ It is associated with the resistance of EGFRTKIs. In the future, it can be a potential agent to deal with resistance of EGFR-TKIs.

\section{Other cancers}

Acute myeloid leukemia is a common hematologic cancer with genetically heterogeneous. It was found that overexpression of GAS5 was associated with shorter overall survival. $^{72}$ In mantle cell lymphoma, targeting the control of GAS5 may significantly improve survival. ${ }^{73}$

The expression of GAS5 was downregulated in melanoma tissue compared with normal tissues. It was reported that expression of $G A S 5$ was related to distant metastases and TNM stage in melanoma by regulating miR-17 transcription. In the future, GAS5 may be a potential target for the treatment of melanoma. ${ }^{49}$

GAS5 was proved to be downregulated in glioma. It was reported that GAS5 can suppress glioma stem cells (GSCs) and further promote apoptosis. ${ }^{46}$ The lower expression of GAS5 was significantly related to increased rate of death, recurrence and progression. ${ }^{74}$

Osteosarcoma is a common malignancy with a high incidence of death in children and young adults. The expression of $G A S 5$ was found significantly decreased in osteosarcoma tissues and cells. GAS5 can suppress cell growth, proliferation and epithelial-mesenchymal transition in osteosarcoma. ${ }^{75}$

In thyroid cancer, the expression of GAS5 was lower than benign tumor tissues. The expression of GAS5 was significantly related to tumor stage, lymph node metastasis, the multiple cancer foci of thyroid cancer, disease-free survival and overall survival. ${ }^{76}$

In head and neck cancer patients treated with radical chemoradiotherapy (CRT), the expression of GAS5 was lower in patients achieved complete response than those with partial response and progressive disease. Thus, GAS5 can be a prognostic biomarker for head and neck cancer in CRT therapy. ${ }^{25}$

Patients with low expression of GAS5 was reported to have poorer disease-free survival and overall survival than those with higher level in ovarian cancer. And the overexpression 
of GAS5 can suppress ovarian cancer cell proliferation and promote apoptosis. $^{77}$ GAS5 can act as a tumor suppressor lncRNA in endometrial cancer as well. ${ }^{40}$

In patients with cervical cancer, GAS5 expression level was associated with FIGO stage, metastatic parameter, clinical staging and overall survival. The downregulation of $G A S 5$ was proved to enhance cell proliferation and invasion. $^{24,78}$ Moreover, the expression of GAS5 can influence cisplatin resistance in cervical cancer by regulating the phosphorylation of Akt. ${ }^{79}$

\section{Challenges and future perspectives}

Cancer is a major health issue often associated with gene mutation. It is increasingly acknowledged that not only the change of the protein-coding genes but also non-proteincoding genes can contribute to different cancers. With lncRNA GAS5 becomes a hot topic, much evidence indicates that IncRNA GAS5 represents a potent tumor suppressor and is aberrantly expressed in various cancers. The suppressive function is involved in multiple pathways including proliferation, metastasis, invasion and CSCs. LncRNA GAS5 can interact with miRNAs and then regulate different genes to regulate the related pathways (Table 1). It has been found that LncRNA GAS5 is lower expressed in a variety of cancers including $\mathrm{CRC}$, breast cancer, gastric cancer, hepatocellular carcinoma, osteosarcoma, esophageal carcinoma and pancreatic cancer. In tumors, lower expression of lncRNA GAS5 is significantly associated with clinicopathological features such as TNM stage, histological grade, tumor size and distant metastasis (Table 1). Moreover, the expression of lncRNA GAS5 can affect the survival and prognosis of some cancers. The exact mechanism of IncRNA GAS5 action is not completely known. The molecular mechanism of lncRNA GAS5 in cancer progression involves in GR, TRIB3, c-Myc, eIF4E, EZH2, TRAIL, CDK6, FGF1, PTEN, ARHI, CXCR4, (AKT)/mTOR, EGFR and NODAL pathway.

However, several challenges exist in the GAS5 field, including the relatively low level in the plasma compared to the normal tissues. Nowadays, the studies of IncRNA GAS5 are still in preclinical stage, the number of cancer patients involved is limited. The precious concentration of lncRNA GAS5 in the serum of cancer patients and healthy patients has not been established. And the challenge here is the standardization of detection methods worldwide. It is unclear whether GAS5 interact with additional chromatinmodifying enzymes through other molecular mechanisms.

GAS5 can also play an important role in non-cancer disease, such as cardiovascular disease, osteoarthritis, type 2 diabetes, inflammatory bowel disease and autoimmune disease. ${ }^{80-84}$ It still needs more studies to discover the real function in various cancers. Future studies to the precise expression of GAS5 in different diseases and disease progression, regression and response to therapies can be conducted to confirm its potential use as the biomarker in diagnosis and response to therapies.

Table I Related miRNAs in various cancers and lower expression of IncGAS5 is associated with clinical features

\begin{tabular}{|c|c|c|}
\hline Cancer type & Related miRNA & Clinicopathological features \\
\hline Colorectal cancer & miR-I03, miR-22I & $\begin{array}{l}\text { Larger tumor size, low histological grade, advanced TNM stage, poor } \\
\text { Prognosis, lymph node metastasis, local recurrence rate, distant metastasis rate }\end{array}$ \\
\hline Cervical cancer & miR-196a, miR-205 & Advanced FIGO stage and metastatic parameter, poorer overall survival \\
\hline Gastric cancer & miR-222, miR-23a & Larger tumor size, advanced pathologic stage, poorer DFS, poorer OS \\
\hline Pancreatic cancer & miR-18Ic-5p & Chemotherapeutic drug resistance, promote tumorigenesis and metastasis \\
\hline Hepatocellular carcinoma & miR-2I & Poor prognosis, differentiation, portal vein \\
\hline Prostate cancer & miR-I03 & Poor outcome \\
\hline Breast cancer & miR-2I & Trastuzumab resistance \\
\hline NSCLC & miR-23a, miR-135b & Advanced TNM stages and larger tumor size, poor tumor differentiation \\
\hline Glioma cancer & miR-196a-5p, miR-222 & - \\
\hline Osteosarcoma & miR-203a & - \\
\hline Melanoma & miR-137 & - \\
\hline Bladder cancer & - & $\begin{array}{l}\text { Higher pathological grades, poor disease free survival, } \\
\text { Enhance the chemotherapy resistance to doxorubicin }\end{array}$ \\
\hline Thyroid cancer & - & Advanced TNM stages, lymph node metastasis, poor DFS, poor OS \\
\hline Head and neck cancer & - & Higher complete response rate \\
\hline
\end{tabular}

Abbreviations: NSCLC, non-small cell lung cancer; DFS, disease free survival; OS, overal survival. 
The potential of GAS5 for cancer diagnosis has been supported by many studies. It can impact the chemotherapy sensitivity in certain cancer and provides a new strategy for overcoming drug resistance. Nowadays, precise medical treatments such as targeted therapies have the advantage of precise specificity and low toxicity. In future studies, lncRNA GAS5 may serve as a new molecular target for the treatment of cancer.

\section{Conclusion}

In conclusion, the discovery of GAS5 has provided new hope to cancer patients. Decreased expression of GAS5 is associated with less proliferation, invasion and metastasis in cancer cells and always predicts advanced TNM stage, high recurrence and poor prognosis in various tumors. We hope to identify novel and sensitive biomarkers and therapeutic targets in cancer patients by understanding the molecular related pathways and clinicopathologic features in different cancers. Future investigation of GAS5 may lead to novel therapeutic strategies.

\section{Acknowledgment}

This work was supported by a grant from the Ph.D. Programs Foundation of Ministry of Education of China (No. 20120101120132)

\section{Disclosure}

\section{References}

1. Siegel RL, Miller KD, Jemal A. Cancer statistics, 2018. CA Cancer J Clin. 2018;68:7-30. doi:10.3322/caac. 21442

2. Spizzo R, Almeida MI, Colombatti A, et al. Long non-coding RNAs and cancer: a new frontier of translational research. Oncogene. 2012;31:4577-4587. doi:10.1038/onc.2011.621

3. Kugel JF, Goodrich JA. Non-coding RNAs: key regulators of mammalian transcription. Trends Biochem Sci. 2012;37:144-151. doi:10.1016/j.tibs.2011.12.003

4. Cho S, Xu J, Sun R, et al. Promoter of lncRNA gene PVT1 is a tumor-suppressor DNA boundary element. Cell. 2018;173: 1398-1412. doi:10.1016/j.cell.2018.03.068

5. Hosono Y, Niknafs Y, Prensner J, et al. Oncogenic role of THOR, a conserved cancer/testis long non-coding RNA. Cell. 2017;171:1559-1572. doi:10.1016/j.cell.2017.11.040.

6. Abraham J, Meltzer S. Long noncoding RNAs in the pathogenesis of Barrett's esophagus and esophageal carcinoma. Gastroenterology. 2017;153:27-34. doi:10.1053/j.gastro.2017.04.046

7. Kim H, Eoh K, Kim L, et al. The long noncoding RNA HOXA11 antisense induces tumor progression and stemness maintenance in cervical cancer. Oncotarget. 2016;7:83001-83016.

8. Khan M, Xiang S, Song Z, et al. The p53-inducible long noncoding RNA TRINGS protects cancer cells from necrosis under glucose starvation. EMBO J. 2017;36:3483-3500.

9. Zhang D, Zhang G, Hu X, et al. Oncogenic RAS regulates long noncoding RNA in human cancer. Cancer Res. 2017;77:3745-3757.
10. Sun D, Yu Z, Fang X, et al. LncRNA GAS5 inhibits microglial M2 polarization and exacerbates demyelination. EMBO Rep. 2017;18:1801-1816. doi:10.15252/embr.201643668

11. Fang L, Sun J, Pan ZF, et al. Long non-coding RNA NEAT1 promotes hepatocellular carcinoma cell proliferation through the regulation of miR-129-5p-VCP-I kappa B. Am J Physiol Gastrointest Liver Physiol. 2017;313:G150-G156. doi:10.1152/ajpgi.00426.2016

12. Lee DW, Kochenderfer JN, Stetler-Stevenson M, et al. T cells expressing CD19 chimeric antigen receptors for acute lymphoblastic leukaemia in children and young adults: a phase 1 dose-escalation trial. Lancet. 2015;385:517-528. doi:10.1016/S0140-6736(14)61403-3

13. Pickard MR, Mourtada-Maarabouni M, Williams GT. Long non-coding RNA GAS5 regulates apoptosis in prostate cancer cell lines. Biochim Biophys Acta. 2013;1832:1613-1623. doi:10.1016/j. bbadis.2013.05.005

14. Schneider C, Schneider C, King RM, et al. Genes specifically expressed at growth arrest of mammalian cells. Cell. 1988;54:787-793.

15. Zhou Y, Wu K, Jiang J, et al. Integrative analysis reveals enhanced regulatory effects of human long intergenic non-coding RNAs in lung adenocarcinoma. J Genet Genomics. 2015;42:423-436. doi:10.1016/ j.jgg.2015.07.001

16. Han L, Ma P, Liu SM, et al. Circulating long noncoding RNA GAS5 as a potential biomarker in breast cancer for assessing the surgical effects. Tumour Biol. 2016;37:6847-6854. doi:10.1007/s13277-0154568-7

17. Sun M, Jin FY, Xia R, et al. Decreased expression of long noncoding RNA GAS5 indicates a poor prognosis and promotes cell proliferation in gastric cancer. BMC Cancer. 2014;14:319. doi:10.1186/14712407-14-319

18. Mourtada-Maarabouni M, Pickard MR, Hedge VL, et al. GAS5, a non-protein-coding RNA, controls apoptosis and is downregulated in breast cancer. Oncogene. 2009;28:195-208. doi:10.1038/onc.2008.373

19. Chen CY, Chen CC, Shieh TM, et al. Corylin suppresses hepatocellular carcinoma progression via the inhibition of epithelial-mesenchymal transition. Mediated by Long Noncoding RNA GAS5. Int J Mol Sci 2018;19:380.

20. $\mathrm{Cz} \mathrm{D}, \mathrm{Xf} \mathrm{G}, \mathrm{Gl} \mathrm{W}$, et al. High glucose contributes to the proliferation and migration of non-small cell lung cancer cells via GAS5-TRIB3 axis. Int $J$ Mol Sci. 2018;38(2):BSR20171014. doi:10.1042/ BSR20171014

21. Lu X, Fang Y, Wang Z, et al. Downregulation of gas5 increases pancreatic cancer cell proliferation by regulating CDK6. Cell Tissue Res. 2013;354:891-896. doi:10.1007/s00441-013-1711-x

22. Guo X, Deng K, Wang H, et al. GAS5 inhibits gastric cancer cell proliferation partly by modulating CDK6. Oncol Res Treat. 2015;38:362-366. doi:10.1159/000433499

23. Tan Q, Zuo J, Qiu S, et al. Identification of circulating long non-coding RNA GAS5 as a potential biomarker for non-small cell lung cancer diagnosisnon-small cell lung cancer, long non-coding RNA, plasma, GAS5, biomarker. Int J Oncol. 2017;50:1729-1738. doi:10.3892/ijo.2017.3925

24. Cao S, Liu W, Li F, et al. Decreased expression of lncRNA GAS5 predicts a poor prognosis in cervical cancer. Int J Clin Exp Pathol. 2014;7:6776-6783.

25. Fayda M, Isin M, Tambas M, et al. Do circulating long non-coding RNAs (lncRNAs) (LincRNA-p21, GAS 5, HOTAIR) predict the treatment response in patients with head and neck cancer treated with chemoradiotherapy? Tumour Biol. 2016;37:3969-3978. doi:10.1007/s13277-015-4189-1

26. Amaldi F, Pierandrei-Amaldi P. TOP genes: a translationally controlled class of genes including those coding for ribosomal proteins. Prog Mol Subcell Biol. 1997;18:1-17.

27. Smith CM, Steitz JA. Classification of gas5 as a multi-smallnucleolar-RNA (snoRNA) host gene and a member of the 5'terminal oligopyrimidine gene family reveals common features of snoRNA host genes. Mol Cell Biol. 1998;18:6897-6909. 
28. Wang KC, Chang HY. Molecular mechanisms of long noncoding RNAs. Mol Cell. 2011;43:904-914. doi:10.1016/j.molcel.2011.08.018

29. Marchese FP, Raimondi I, Huarte M. The multidimensional mechanisms of long noncoding RNA function. Genome Biol. 2017;18:206. doi:10.1186/s13059-017-1348-2

30. Sturmberg JP, Bennett JM, Picard M, et al. The trajectory of life. Decreasing physiological network complexity through changing fractal patterns. Front Physiol. 2015;6:169.

31. Franchimont D, Kino T, Galon J, et al. Glucocorticoids and inflammation revisited: the state of the art. Neuroimmunomodulation. 2003;10:247-260. doi:10.1159/000069969

32. Kino T, Hurt DE, Ichijo T, et al. Noncoding RNA gas5 is a growth arrest- and starvation-associated repressor of the glucocorticoid receptor. Sci Signal. 2010;3:ra8. doi:10.1126/scisignal.2000568

33. Wang M, Guo C, Wang L, et al. Diesel exhaust particles induce autophagy and citrullination in Normal Human Bronchial Epithelial cells. Cell Death Dis. 2018;9:238. doi:10.1038/s41419-018-1111-y

34. Toraih EA, Alghamdi SA, El-Wazir A, et al. Dual biomarkers long non-coding RNA GAS5 and microRNA-34a co-expression signature in common solid tumors. PLoS One. 2018;13:e0198231. doi:10.1371/ journal.pone.0198231

35. Hu L, Ye H, Huang G, et al. Long noncoding RNA GAS5 suppresses the migration and invasion of hepatocellular carcinoma cells via miR-21. Tumour Biol. 2016;37:2691-2702. doi:10.1007/s13277-015-4111-X

36. Zhang Z, Zhu Z, Watabe K, et al. Negative regulation of lncRNA GAS5 by miR-21. Cell Death Differ. 2013;20:1558-1568. doi:10.1038/cdd.2013.110

37. Chun-Zhi Z, Lei H, An-Ling Z, et al. The Brustkrebs-Studien.de website for breast cancer patients: user acceptance of a German internet portal offering information on the disease and treatment options, and a clinical trials matching service. BMC Cancer. 2010;10:367. doi:10.1186/1471-2407-10-663

38. Zhao X, Wang P, Liu J, et al. Gas5 exerts tumor-suppressive functions in human glioma cells by targeting miR-222. Mol Ther. 2015;23:1899-1911. doi:10.1038/mt.2015.170

39. Zhang XF, Ye Y, Zhao SJ. LncRNA Gas5 acts as a ceRNA to regulate PTEN expression by sponging miR-222-3p in papillary thyroid carcinoma. Oncotarget. 2018;9:3519-3530. doi:10.18632/ oncotarget. 23336

40. Guo C, Song WQ, Sun P, et al. LncRNA-GAS5 induces PTEN expression through inhibiting miR-103 in endometrial cancer cells. J Biomed Sci. 2015;22:100. doi:10.1186/s12929-015-0213-4

41. Liu X, She Y, Wu H, et al. Discovery and cellular stress pathway analysis of 1,4-naphthoquinone derivatives with novel, highly potent broad-spectrum anticancer activity. J Biomed Sci. 2018;25:18. doi:10.1186/s12929-018-0408-6

42. Cao L, Chen J, Ou B, et al. GAS5 knockdown reduces the chemo-sensitivity of non-small cell lung cancer (NSCLC) cell to cisplatin (DDP) through regulating miR-21/PTEN axis. PLoS One. 2017;93:570-579.

43. Liu L, Meng T, Xh Y, et al. Prognostic and Predictive Value of Long NonCoding RNA GAS5 and mircoRNA-221 in Colorectal Cancer and Their Effects on Colorectal Cancer Cell Proliferation, Migration and Invasion. Cancer Biomark. 2018;22:283-299. doi:10.3233/CBM-171011

44. Xue Y, Ni T, Jiang Y, et al. Long noncoding RNA GAS5 inhibits tumorigenesis and enhances radiosensitivity by suppressing miR-135b expression in non-small cell lung cancer. Sci Rep. 2017;25:1305-1316.

45. Mei Y, Si J, Wang Y, et al. Long noncoding RNA GAS5 suppresses tumorigenesis by inhibiting mir-23a expression in non-small cell lung cancer. Oncol Res. 2017;25:1027-1037. doi:10.3727/ 096504016X14822800040451

46. Zhao X, Liu Y, Zheng J, et al. GAS5 suppresses malignancy of human glioma stem cells via a miR-196a-5p/FOXO1 feedback loop. Biochim Biophys Acta. 2017;1864:1605-1617. doi:10.1016/j. bbamcr.2017.06.020
47. Liu X, Jiao T, Wang Y, et al. Long non-coding RNA GAS5 acts as a molecular sponge to regulate miR-23a in gastric cancer. Int $J$ Exp Pathol. 2016;9:11412-11419

48. Wang Y, Kong D. LncRNA GAS5 represses osteosarcoma cells growth and metastasis via sponging MiR-203a. Cell Physiol Biochem. 2018;45:844-855. doi:10.1159/000487178

49. Bian D, Shi W, Shao Y, et al. Long non-coding RNA GAS5 inhibits tumorigenesis via miR-137 in melanoma. Am $J$ Transl Res. 2017;9:1509-1520.

50. Yang W, Hong L, Xu X, et al. LncRNA GAS5 suppresses the tumorigenesis of cervical cancer by downregulating miR-196a and miR-205. Tumour Biol. 2017;39:1010428317711315. doi:10.1177/ 1010428317711315

51. Fingar DC, Blenis J. Target of rapamycin (TOR): an integrator of nutrient and growth factor signals and coordinator of cell growth and cell cycle progression. Oncogene. 2004;23:3151-3171. doi:10.1038/ sj.onc. 1207542

52. Geng L, Sun B, Gao B, et al. MicroRNA-103 promotes colorectal cancer by targeting tumor suppressor DICER and PTEN. Int $J \mathrm{Mol}$ Sci. 2014;15:8458-8472. doi:10.3390/ijms 15058458

53. Xue D, Zhou C, Lu H, et al. LncRNA GAS5 inhibits proliferation and progression of prostate cancer by targeting miR-103 through AKT/mTOR signaling pathway. Tumour Biol. 2016;37:16187-16197. doi:10.1007/s13277-016-5429-8

54. Li Y, Gu J, Lu H. The GAS5/miR-222 axis regulates proliferation of gastric cancer cells through the PTEN/Akt/mTOR pathway. Dig Dis Sci. 2017;62:3426-3437. doi:10.1007/s10620-017-4831-4

55. Pei J, Wang B. Notch-1 promotes breast cancer cells proliferation by regulating LncRNA GAS5. Int $J$ Clin Exp Med. 2015;8:14464-14471.

56. Dong S, Qu X, Li W, et al. The long non-coding RNA, GAS5, enhances gefitinib-induced cell death in innate EGFR tyrosine kinase inhibitor-resistant lung adenocarcinoma cells with wide-type EGFR via downregulation of the IGF-1R expression. J Hematol Oncol. 2015;8:43. doi:10.1186/s13045-015-0140-6

57. Xu C, Zhang Y, Wang Q, et al. Long non-coding RNA GAS5 controls human embryonic stem cell self-renewal by maintaining NODAL signalling. Nat Commun. 2016;7:13287. doi:10.1038/ ncomms 13287

58. Gao ZQ, Wang JF, Chen DH, et al. Long non-coding RNA GAS5 antagonizes the chemoresistance of pancreatic cancer cells through down-regulation of miR-181c-5p. Biomed Pharmacother. 2018;97:809-817. doi:10.1016/j.biopha.2017.10.157

59. Yin D, He X, Zhang E, et al. Association of FoxP3 rs3761548 polymorphism with susceptibility to colorectal cancer in the Chinese population. Med Oncol. 2014;31:253. doi:10.1007/s12032014-0374-0

60. Li J, Wang Y, Zhang CG, et al. Effect of long non-coding RNA Gas5 on proliferation, migration, invasion and apoptosis of colorectal cancer HT-29 cell line. Cancer Cell Int. 2018;18:4. doi:10.1186/s12935017-0478-7

61. Yang Y, Shen Z, Yan Y, et al. Long non-coding RNA GAS5 inhibits cell proliferation, induces G0/G1 arrest and apoptosis, and functions as a prognostic marker in colorectal cancer. Oncol Lett. 2014;31:3151-3158. doi:10.3892/ol.2017.5841

62. Gao Z-Q, Wang J-F, Chen D-H, et al. Long non-coding RNA GAS5 suppresses pancreatic cancer metastasis through modulating miR-325p/PTEN axis. Cell Biosci. 2017;7:66. doi:10.1186/s13578-017-0192-0

63. Chang L, Li C, Lan T, et al. Decreased expression of long non-coding RNA GAS5 indicates a poor prognosis and promotes cell proliferation and invasion in hepatocellular carcinoma by regulating vimentin. Mol Med Rep. 2016;13:1541-1550. doi:10.3892/mmr.2015.4716

64. Wang K, Li J, Xiong G, et al. Negative regulation of IncRNA GAS5 by miR-196a inhibits esophageal squamous cell carcinoma growth. Biochem Biophys Res Commun. 2018;495:1151-1157. doi:10.1016/j. bbrc.2017.11.119 
65. Li W, Zhao W, Lu Z, et al. Long non-coding RNA GAS5 promotes proliferation, migration and invasion by regulation of miR-301a in esophageal cancer. Oncol Res. 2018;26(8):1285-1294.

66. Renganathan A, Kresoja-Rakic J, Echeverry N, et al. GAS5 long non-coding RNA in malignant pleural mesothelioma. Mol Cancer. 2014;13:119. doi:10.1186/1476-4598-13-119

67. Qiao H-P, Gao W-S, Huo J-X, Yang Z-S. Long non-coding RNA GAS5 functions as a tumor suppressor in renal cell carcinoma. Asian Pac J Cancer Prev. 2013;14:1077-1082.

68. Zhou S, Wang J, Zhang Z. An emerging understanding of long noncoding RNAs in kidney cancer. $J$ Cancer Res Clin Oncol. 2014;140:1989-1995. doi:10.1007/s00432-014-1699-y

69. Zhang H, Guo Y, Song Y, Shang C. Long noncoding RNA GAS5 inhibits malignant proliferation and chemotherapy resistance to doxorubicin in bladder transitional cell carcinoma. Cancer Chemother Pharmacol. 2017;79:49-55. doi:10.1007/s00280-0163194-4

70. Pickard MR, Williams GT. Regulation of apoptosis by long non-coding RNA GAS5 in breast cancer cells: implications for chemotherapy. Breast Cancer Res Treat. 2014;145:359-370.

71. Nagini S. Breast cancer: current molecular therapeutic targets and new players. Anticancer Agents Med Chem. 2017;17:152-163.

72. Yan H, Zhang DY, Li X, et al. Long non-coding RNA GAS5 polymorphism predicts a poor prognosis of acute myeloid leukemia in Chinese patients via affecting hematopoietic reconstitution. Leuk Lymphoma. 2017;58:1948-1957.

73. Mourtada-Maarabouni M, Williams GT. Role of GAS5 noncoding RNA in mediating the effects of rapamycin and its analogues on mantle cell lymphoma cells. Clin Lymphoma Myeloma Leuk. 2014; $14: 468-473$.

74. Shen J, Hodges TR, Song R, et al. Serum HOTAIR and GAS5 levels as predictors of survival in patients with glioblastoma. Mol Carcinog. 2018;57:137-141.
75. Ye K, Wang S, Zhang H, et al. Long noncoding RNA GAS5 suppresses cell growth and epithelial-mesenchymal transition in osteosarcoma by regulating the miR-221/ARHI pathway. $J$ Cell Biochem. 2017;118:4772-4781.

76. Guo LJ, Zhang S, Gao B, et al. Low expression of long non-coding RNA GAS5 is associated with poor prognosis of patients with thyroid cancer. Exp Mol Pathol. 2017;102:500-504.

77. Li J, Huang H, Li Y, et al. Decreased expression of long non-coding RNA GAS5 promotes cell proliferation, migration and invasion, and indicates a poor prognosis in ovarian cancer. Oncol Rep. 2016;36:3241-3250

78. Li Y, Wan YP, Bai Y. Correlation between long strand non-coding RNA GASS expression and prognosis of cervical cancer patients. Eur Rev Med Pharmacol Sci. 2018;22:943-949.

79. Wen Q, Liu Y, Lyu H, et al. Long noncoding RNA GAS5, which acts as a tumor suppressor via microRNA 21, regulates cisplatin resistance expression in cervical cancer. Int $J$ Gynecol Cancer. 2017;27:1096-1108.

80. Simion V, Haemmig S, Feinberg MW. LncRNAs in vascular biology and disease. Vascul Pharmacol. 2018.

81. Xing D, Liang JQ, Li Y, et al. Identification of long noncoding RNA associated with osteoarthritis in humans. Orthop Surg. 2014;6:288-293.

82. Qi M, Zhou Q, Zeng W, et al. Analysis of long non-coding RNA expression of lymphatic endothelial cells in response to type 2 diabetes. Cell Physiol Biochem. 2017;41:466-474.

83. Lucafò M, Di Silvestre A, Romano M, et al. Role of the long non-coding rna growth arrest-specific 5 in glucocorticoid response in children with inflammatory bowel disease. Basic Clin Pharmacol Toxicol. 2018;122:87-93.

84. Mourtada-Maarabouni M, Hedge VL, Kirkham L, et al. Growth arrest in human T-cells is controlled by the non-coding RNA growth-arrestspecific transcript 5 (GAS5). J Cell Sci. 2008;121:939-946.

\section{Publish your work in this journal}

Cancer Management and Research is an international, peer-reviewed open access journal focusing on cancer research and the optimal use of preventative and integrated treatment interventions to achieve improved outcomes, enhanced survival and quality of life for the cancer patient.
The manuscript management system is completely online and includes a very quick and fair peer-review system, which is all easy to use. Visit http://www.dovepress.com/testimonials.php to read real quotes from published authors. 